\title{
MODULE STRUCTURE OF CERTAIN INDUCED REPRESENTATIONS OF COMPACT LIE GROUPS
}

\author{
BY
}

\author{
E. JAMES FUNDERBURK
}

\begin{abstract}
Let $G$ be a compact connected Lie group and assume a choice of maximal torus and positive roots has been made. Given a dominant weight $\lambda$, the Borel-Weil Theorem shows how to construct a holomorphic line bundle on whose sections $G$ acts so that the holomorphic sections provide a realization of the irreducible representation of $G$ with highest weight $\lambda$. This paper studies the $G$-module structure of the space $\Gamma$ of square integrable sections of the Borel-Weil line bundle. It is found that $\Gamma=\lim _{n \rightarrow \infty} \Gamma(n)$, where $\Gamma(n) \subset \Gamma(n+1) \subset \Gamma$ and $\Gamma(n)$ is isomorphic, as $G$-module, to

$$
V(\lambda+n \lambda) \otimes V\left(n \lambda^{*}\right) \text {, }
$$
\end{abstract}

where $V(\mu)$ denotes the irreducible representation of highest weight $\mu$, ' + ' is the Cartan semigroup operation, and '*' is the contragredient operation. Similar formulas hold for powers of the Borel-Weil line bundle.

1. Introduction. In the formulation of quantum mechanics proposed by J.-M. Souriau (see [14]) one is led, given a Lie group $G$, to the study of certain homogeneous Hermitian line bundles with connection whose bases have a symplectic structure determined as the curvature of the given connection. It is a natural question to ask the $G$-module structure of the sections of such homogeneous line bundles. For the case when $G$ is a compact connected Lie group, this paper presents an answer to this question. In the solution presented one also obtains the $G$-module structure of square-integrable functions on both the total space and base of the associated principal bundle.

The answer obtained may be expressed as follows. Let $T$ be a maximal torus in the compact connected Lie group $G$ and $\Lambda^{+}$the set of dominant weights for $G$ with respect to a fixed choice of positive roots. Set theoretically we view $\Lambda^{+}$as a certain set of linear functions on the Lie algebra of $G$, closed under addition and containing the zero linear functional. The Cartan theory of highest weights presents an identification of $\Lambda^{+}$with $\hat{G}$, the set of, necessarily finite-dimensional and unitary, irreducible representations of $G$.

Let $\mathcal{S}$ denote the set of all functions from $\hat{G}$ to the nonnegative integers, $\mathbf{Z}^{+}$, and let $\Re$ denote the set of unitary equivalence classes of completely

Received by the editors November 6, 1975.

AMS (MOS) subject classifications (1970). Primary 17B10, 43A85; Secondary 22E45, $17 \mathrm{~B} 20$.

Key words and phrases. Compact connected Lie group, highest weight, lowest weight, oppositıon involution, Cartan semigroup, tensor product, cyclic representation, Borel-Weil realization. 
continuous unitary representations of $G$. Then, there is a bijection ch: $\Re \rightarrow \mathcal{R}$, such that $\operatorname{ch}(\rho)(\lambda)$ is the multiplicity of $\lambda$ as a subrepresentation of $\rho$, for $\rho$ in $R, \lambda$ in $G$. Thus the function $\operatorname{ch}(\rho)$ determines the $G$-module structure of $\rho$. We refer to $\operatorname{ch}(\rho)$ as the formal character of $\rho$.

The elements of $R$ whose formal characters we will determine may be described as follows. Fix $\lambda$ in $\hat{G}$ and denote by $\lambda^{*}$ in $\hat{G}$ the representation contragredient to $\lambda$. Let $K$ denote the isotropy group of $\lambda^{*}$ in $G$ with respect to the contragredient adjoint action of $G$. Let $R$ and $L$ denote the right and left regular representations of $G$ in $L^{2}(G)$. Let $\Gamma$ and $\Gamma_{k}$ (for $k$ in $Z$ ) denote the elements of $\mathcal{R}$ which are subrepresentations of $L$ determined by the following requirements on $f$ in $L^{2}(G)$ :

$f \in \Gamma$ iff $R(x) f=f, x \in K_{0}$,

$f \in \Gamma_{k}$ iff $R(x) f=\chi(x)^{k} f, x \in K$.

Here $\chi: K \rightarrow S^{1}$ is the homomorphism determined by $\lambda^{*}\left(\chi(\exp X)=e^{\lambda^{*}(x)}\right.$, for $X$ in the Lie algebra of $K$ ), and $K_{0}$ is the kernel of $\chi$. It is clear that $\Gamma_{k} \subset \Gamma$ for all $k$, and one may show $\Gamma=\bigoplus_{k \in \mathbf{Z}} \Gamma_{k}$, a direct sum in $\Re . \Gamma_{1}$ may be interpreted as the $L^{2}$ sections of a homogeneous Hermitian line bundle with connection $E \rightarrow M$ determined by $\lambda^{*}, \Gamma_{0}$ as $L^{2}(M)$, and $\Gamma$ as $L^{2}(P)$, $S^{1} \rightarrow P \rightarrow M$ being the associated principal bundle to $E \rightarrow M$.

We express $\operatorname{ch}\left(\Gamma_{k}\right)$ as the limit of a certain bounded increasing sequence in $\mathcal{S}(f<g$ in $\mathcal{S}$ iff $f(\lambda)<g(\lambda)$ for all $\lambda$ in $\hat{G}$; a subset $S$ of $\mathcal{S}$ is bounded iff $\{f(\lambda) \mid f \in \mathcal{S}, \lambda \in \hat{G}\}$ is a bounded set in $\mathbf{Z}^{+}$); obviously such a sequence has a unique point-wise defined limit in $\mathcal{S}$. It is shown that

$$
\left\{\operatorname{ch}\left(\mu+n \nu \otimes n \nu^{*}\right)\right\}_{n=0}^{\infty} \text { and }\left\{\operatorname{ch}\left(n \nu \otimes \mu+n \nu^{*}\right)\right\}_{n=0}^{\infty}
$$

are bounded increasing sequences in $\mathcal{S}$, for $\mu, \nu$ in $\Lambda^{+}$. Then for $k$ in $\mathbf{Z}^{+}$, our main result states

$\operatorname{ch}\left(\Gamma_{k}\right)=\lim _{n \rightarrow \infty} \operatorname{ch}\left(k \lambda+n \lambda \otimes n \lambda^{*}\right), \quad \operatorname{ch}\left(\Gamma_{-k}\right)=\lim _{n \rightarrow \infty} \operatorname{ch}\left(n \lambda \otimes k \lambda^{*}+n \lambda^{*}\right)$.

In particular

$$
\begin{aligned}
& \operatorname{ch}\left(\Gamma_{1}\right)=\lim _{n \rightarrow \infty} \operatorname{ch}\left((\lambda+n \lambda) \otimes n \lambda^{*}\right), \quad \operatorname{ch}\left(\Gamma_{0}\right)=\lim _{n \rightarrow \infty} \operatorname{ch}\left(n \lambda \otimes n \lambda^{*}\right) \\
& \operatorname{ch}(\Gamma)=\sum_{k=0}^{\infty} \lim _{n \rightarrow \infty}\left(\operatorname{ch}\left((k \lambda+n \lambda) \otimes n \lambda^{*}\right)+\operatorname{ch}\left(n \lambda \otimes\left(k \lambda^{*}+n \lambda^{*}\right)\right)\right)
\end{aligned}
$$

The basic idea involved in establishing the above formulas may be referred to as the Borel-Weil realizations of elements of $\hat{G}$. For $\lambda$ in $\hat{G}=\Lambda^{+}$, consider the systems of differential equations:

$$
\begin{gathered}
\left(R(X)-\lambda^{*}(X)\right) f=0, \\
(L(X)-\lambda(X)) f=0,
\end{gathered}
$$


for $X$ a positive root vector or an element of the Lie algebra of $T$. Let $\mathscr{B}_{\lambda}$ denote the simultaneous solutions to (1); and $\mathscr{T}_{\lambda}^{0}$ the simultaneous solutions to (1) and (2). Then, the subrepresentation of $L$ in $\mathscr{B}_{\lambda}$ is a representative of $\lambda$ in $\hat{G}$ and $\mathscr{S}_{\lambda}$ is the highest weight space; we take this statement to be the Borel-Weil Theorem, and refer to $B_{\lambda}$ as the Borel-Weil realization of $\lambda$. One has the relations $B_{\lambda+\nu}=B_{\lambda} B_{\nu}$ (equality of sets, $B_{\lambda} B_{\nu}$ is the complex span of point-wise defined products $f g$ with $f$ in $B_{\nu}, g$ in $B_{\nu}$ ). $\bar{B}_{\nu}$ is isomorphic to $B_{\nu^{*}}$ ( $\bar{B}_{\nu}$ is the set of $\bar{f}$ with $f$ in $B_{\nu}$ ) and the multiplication map $B_{\lambda} \otimes B_{\nu} \rightarrow B_{\lambda} \bar{B}_{\nu}$ a (nonunitary) $G$-module equivalence; thus $B_{\lambda} \bar{B}_{\nu^{*}}$ as a subrepresentation of $L$ is isomorphic to the tensor product $\lambda \otimes \nu$.

Borel-Weil realizations are related to the original question by using the Stone-Weierstrass Theorem to show $\sum_{p, q \in \mathbf{Z}^{+}} \mathscr{B}_{p \lambda} \overline{\mathscr{G}}_{q \lambda}$ is dense in $\Gamma=L^{2}(P)$.

For certain special cases we determine $\operatorname{ch}\left(\Gamma_{1}\right)$ explicitly by working out the tensor product limits of our general expression for $\operatorname{ch}\left(\Gamma_{1}\right)$. There is a generally applicable theoretical formula for the Clebsch-Gordon series for the tensor product of two irreducible representations (Steinberg's formula). When $\lambda$ is regular, multiplicity formulas, such as those of Kostant and of Freudenthal, are of practical use in computing $\operatorname{ch}\left(\Gamma_{k}\right)$.

The author expresses his appreciation to Joel W. Robbin for his lectures on the Souriau-Kostant approach to quantum mechanics and representation theory of Lie groups; these lectures and ensuing conversations made it possible to consider the questions of this paper.

2. Compact connected Lie groups. Throughout this paper $G$ denotes a compact connected Lie group, $\mathcal{G}$ its Lie algebra and $\mathcal{G}_{\mathbf{C}}$ the complexification of $\mathcal{G}$.

2.1. Background on completely continuous representations. $C(G)\left(C^{\infty}(G)\right)$ denotes the complex vector space of continuous (smooth) functions on $G$. $L^{2}(G)$ denotes the Hilbert space of functions on $G$ that are square-integrable with respect to the normalized bi-invariant Haar measure $d x$ on $G$.

We denote by $\mathcal{R}$ (respectively, $\hat{G}$ ) the set of unitary equivalence classes of completely continuous (respectively, irreducible) unitary representations of $G$. $L$ and $R$, the left-regular and right-regular representations of $G$ in $L^{2}(G)$, are (equal) elements of $\mathcal{R}$ (see 2.6.2 and 2.8.2 of [17]).

If $\rho$ is a unitary representation we may denote by $V^{\rho}$ the Hilbert space in which $\rho$ acts by unitary operators. For $\rho$ in $\hat{G}, V^{\rho}$ is a finite-dimensional vector space (see 2.6.3 of [17]) of dimension $d_{\rho}$.

For $\rho$ in $\hat{G}$ we assume that an orthonormal basis $\left\{v_{i}^{\rho}\right\}_{i=1, \ldots, d_{\rho}}$ has been chosen for $V^{\rho}$; in (2.4) a certain choice of basis will be made. Define $\rho_{i j} \in C(G)$ by $\rho_{i j}(a)=\left\{\rho(a) v_{j}^{\rho}, v_{i}^{\rho}\right\}, a \in G, 1 \leqslant i, j \leqslant d_{\rho}$. The Peter-Weyl Theorem [5, p. 203] asserts the density in $L^{2}(G)$ of $\left\{\rho_{i j} \mid \rho \in G, 1 \leqslant i, j \leqslant d_{\rho}\right\}$. Defining $E^{i, j, \rho}$ in $\operatorname{End}\left(V^{\rho}\right)$ (the set of linear maps from $V^{\rho}$ to $V^{\rho}$ ) by 


$$
E^{i j, \rho} v_{k}^{\rho}=\delta_{j k} v_{i}^{\rho}, \quad 1 \leqslant i, j, k \leqslant d_{\rho},
$$

one has $\rho(a)=\sum_{i, j} \rho_{i j}(a) E^{i, j, \rho}, a \in G$.

Declaring $\left\{d_{\rho}^{-i / 2} E^{i, j, \rho}: 1 \leqslant i, j \leqslant d_{\rho}\right\}$ to be an orthonormal set imposes a Hilbert space structure on $\operatorname{End}\left(V^{\rho}\right)$. One may form the Hilbert space direct sum $\mathcal{E}$ of the $\operatorname{End}\left(V^{\rho}\right)$ for $\rho$ in $\hat{G}$. For $A$ in $\mathcal{E}$ and $\rho$ in $\hat{G}, A_{\rho}$ denotes the component of $A$ in $\operatorname{End}\left(V^{\rho}\right)$.

The Fourier transform $\mathscr{F}: L^{2}(G) \rightarrow \mathcal{E}$ and the inverse Fourier transform $\mathscr{F}^{*}: \mathcal{E} \rightarrow L^{2}(G)$ are mutually inverse isometries such that for $f$ in $C(G), \rho$ in $\hat{G}, A$ in $\operatorname{End}\left(V^{\rho}\right), y$ in $G$,

$$
\mathscr{F}(f)_{\rho}=\int_{G} f(x) \rho(x) d x, \quad \mathscr{F}^{*}(A)(y)=d_{\rho} \operatorname{tr}\left(A \rho\left(y^{-1}\right)\right) .
$$

If $\rho$ is in $\Re$ and $\lambda$ is in $\hat{G}$, then the finite dimension of the space of all operators that intertwine $\lambda$ and $\rho$ we denote by $\operatorname{ch}(\rho)(\lambda)$. Thus, ch sets up a one-to-one correspondence between $\Re$ and the set $\mathbb{S}$ of functions from $\hat{G}$ to $\mathbf{Z}^{+}$. We call $\operatorname{ch}(o)$ the formal character of the completely continuous representation $\rho$.

2.2. Cartan semigroup. Let $\rho$ be a unitary representation of $G$. If $v$ is a smooth vector we set

$$
\rho(X) v=\lim _{t \rightarrow 0}(\rho(\exp (t X)) v-v) / t, \quad X \in \mathcal{G} .
$$

From now on in $\$ 2$ all representations are assumed to be finite-dimensional unitary. For a representation $\rho$ of $G$ we denote also by $\rho$ the associated Lie algebra homomorphism $\mathcal{G} \rightarrow \operatorname{End}\left(V^{\rho}\right)$ or its complex linear extension $\mathcal{G}_{\mathbf{C}} \rightarrow$ $\operatorname{End}\left(V^{\rho}\right)$.

We assume fixed a maximal torus $T$ of $G$ with Lie algebra $T$. $\mathcal{Z}$ denotes the complexification of the Lie algebra of the center of $G, E=\left[{ }_{C},{ }_{C}\right]$, $\mathcal{H}=\mathcal{T}_{\mathbf{c}} \cap \mathcal{L}$.

The complexified adjoint representation of $G$ in $\mathcal{G}_{\mathbf{C}}$ is denoted by ad, its contragredient by ad*. We assume fixed a positive definite inner product on $\mathcal{G}$ extended to Hermitian inner product on $\mathcal{G}_{\mathbf{C}}$ and dualized to one on $\mathcal{S}_{\mathbf{C}}^{*}$ which (see 5.6.1 of [17]) is $\operatorname{ad}(G)$ invariant, is equal to the negative Killing form on $\mathcal{L} \cap \mathcal{G}$, and renders $\mathcal{L}$ and $\mathscr{Z}$ perpendicular. By means of the splittings $\mathcal{G}_{\mathbf{C}}=\mathscr{T}_{\mathbf{C}} \oplus \mathcal{T}_{\mathbf{C}}^{\perp}, \mathscr{T}_{\mathbf{C}}=\mathscr{H}_{\mathcal{C}} \oplus \mathscr{Z}$, we consider $\mathscr{T}_{\mathbf{C}}^{*}$ and $\mathfrak{H}^{*}$ as subspaces of $\mathcal{G}_{\mathbf{C}}^{*}$.

If $\rho$ is a representation of $G$ and $\lambda$ is in $\sigma_{C}^{*}$, set $V_{\lambda}^{\rho}=\left\{v \in V^{\rho} \mid \rho(\exp (H)) v\right.$ $=e^{\lambda(H)} v$ if $\left.H \in \mathcal{T}\right\}$. If $V_{\lambda}^{\rho} \neq(0)$ we say that $\lambda$ is a weight of $\rho, V_{\lambda}^{\rho}$ is the $\lambda$ weight space of $\rho$ and nonzero elements of $V_{\lambda}^{\rho}$ are weight vectors of $\rho$ of weight $\lambda ; \Lambda(\rho)$ is the set of weights of $\rho$.

The root system $\Phi$ of $G$ with respect to $T$ may be defined as $\Lambda(\mathrm{ad})-\{0\}$. We assume a fixed set $\Phi^{+}$of positive roots has been chosen, and set 
$\Phi^{-}=-\Phi^{+}$and denote by $\Re^{+}\left(\Re^{-}\right)$the vector space sum of the positive (negative) root spaces; $\mathcal{G}_{\alpha}$ denotes the $\alpha$ weight space of ad, for $\alpha \in \Phi$.

Let $\Lambda^{+}$denote the set of dominant weights of $G$ (with respect to $T, \Phi^{+}$). As a subset of the additive vector space $\mathscr{T}_{\mathbf{C}}^{*}, \Lambda^{+}$is an abelian semigroup with identity. Since identified with $\Lambda^{+} \mathrm{v}$ ia the correspondence of $\rho$ in $\hat{G}$ with its highest weight $\lambda_{\rho}$ in $\Lambda^{+}, \hat{G}$ is an abelian semigroup, called the Cartan semigroup.

2.3. Weyl group; opposition involution. The Weyl group $W$ of $G$ (with respect to $T$ ) may be defined as $N(T) / T$, where $N(T)=\left\{a \in G \mid n T n^{-1}=T\right\}$, or as the subgroup of linear automorphisms of $\mathcal{T}_{\mathbf{C}}^{*}$ generated by the reflections $\left\{\sigma_{\alpha} \mid \alpha \in \Phi\right\}$, where

$$
\sigma_{\alpha}(\lambda)=\lambda-2(\{\lambda, \alpha\} \alpha /\{\alpha, \alpha\}),
$$

for $\alpha, \lambda$ in $\mathscr{T}_{\mathbf{C}}^{*}$. If $\operatorname{ad}^{*}(n)$, for $n$ in $N(T)$, induces the automorphism $w$ of $\mathcal{T}_{\mathbf{C}}^{*}$, we may write $w=n T$.

There is a unique element $w_{0}$ in $W$ with $w_{0} \Phi^{+}=\Phi^{-}$; since $w_{0}^{2}$ is the identity, $w_{0}$ is called the opposition involution (with respect to $T, \Phi^{+}$). We assume $n_{0}$ in $N(T)$ chosen with $w_{0}=n_{0} T$.

2.4. Lowest weight. For each $\rho$ in $\hat{G}$ there is unique $\lambda_{\rho}^{-}$in $-\Lambda^{+}$so that $\lambda_{\rho}^{-} \in \Lambda(\rho)$ but, for $\alpha \in \Phi^{-}, \lambda_{\rho}^{-}+\alpha \notin \Lambda(\rho) ; \lambda_{\rho}^{-}$is called the lowest weight of $\rho$ and any nonzero element of the one-dimensional space $V_{\lambda_{\rho}^{-}}^{\rho}$ is called a lowest weight vector.

Proposition (2.4.1). For $\rho$ in $\hat{G}, w_{0}\left(\lambda_{\rho}\right)=\lambda_{\rho}^{-}=-\lambda_{\rho^{*}}$ and $\rho\left(n_{0}^{\ell}\right) V_{\lambda_{\rho}^{\rho}}^{\rho}=V_{\lambda_{\rho}^{-}}^{\rho}$, for $\varepsilon= \pm 1$. Here $\rho^{*}$ is the representation of $G$ in $\left(V^{\rho}\right)^{*}$ contragredient to $\rho$.

Proof. Choose $\alpha$ in $\Phi^{+}, X_{-\alpha}$ in $\mathcal{G}_{-\alpha}$, and $v$ in $V_{\lambda_{\rho}}^{\rho}$. As $\operatorname{ad}\left(n_{0}\right) \mathcal{Q}_{-\alpha} \subset$ $\mathcal{G}_{w_{0}(-\alpha)} \subset n^{+}$, one has $\rho\left(X_{-\alpha}\right) \rho\left(n_{0}^{-1}\right) v=\rho\left(n_{0}^{-1}\right) \rho\left(\operatorname{ad}\left(n_{0}\right) X_{-\alpha}\right) v=0$. It follows that $V_{\lambda_{\rho}^{-}}^{\rho}=\rho\left(n_{0}^{-1}\right) V_{\lambda_{\rho}}^{\rho}=V_{w_{0}(\lambda \rho)}^{\rho}$, so $\lambda_{\rho}^{-}=w_{0}(\lambda \rho)$. From $\Lambda\left(\rho^{*}\right)=$ $-\Lambda(\rho)$ we see that $-\lambda_{\rho^{*}}-\alpha$ is not in $\Lambda(\rho)$ for $\alpha$ in $\Phi^{+}$and that $-\lambda_{\rho^{*}}$ is in $\Lambda(\rho)$. By the uniqueness of $\lambda_{\rho}^{-},-\lambda_{\rho^{*}}=\lambda_{\rho}^{-}$. Q.E.D.

From now on in this paper we assume the Cartan identification of $\hat{G}$ with $\Lambda^{+}$. Frequently elements of $\hat{G}$ are denoted by $\lambda$ in $\Lambda^{+}$; sometimes $\rho_{\lambda}(a)$ may be written in place of $\lambda(a)$, for $a$ in $G$.

Suppose $\lambda$ in $\Lambda^{+}=\hat{G}$ is chosen. We choose once and for all, an orthonormal basis $\left\{v_{i}^{\lambda}\right\}_{i=1, \ldots, d(\lambda)}$ of $V^{\lambda}$ consisting of weight vectors and enumerated in such a way that $v_{1}^{\lambda}$ is of weight $\lambda$ and $v_{d(\lambda)}^{\lambda}$ is of weight $-\lambda^{*}$, where $\lambda^{*}$ is the element of $\Lambda^{+}$corresponding to the contragredient of $\lambda$. We denote by $\left\{\phi_{i}^{\lambda}\right\}_{i=1, \ldots, d(\lambda)}$ the basis of $V^{\lambda^{*}}$ dual to $\left\{v_{i}^{\lambda}\right\}_{i=1, \ldots, d(\lambda)}$; thus $\phi_{d(\lambda)}$ is of weight $\lambda^{*}$ and $\phi_{1}^{\lambda}$ of weight $-\lambda$. We may further assume that $v_{d(\lambda)}^{\lambda}=\lambda\left(n_{0}\right) v_{1}^{\lambda}$ and define $\zeta_{0} \in S^{1}$ by $\lambda\left(n_{0}^{-1}\right) v_{1}^{\lambda}=\zeta_{0} v_{d(\lambda)}^{\lambda}$.

For later use we introduce the notations $f_{i}^{\lambda}$ for $\bar{\lambda}_{i d(\lambda)}$ and $\mathscr{B}_{\lambda}$ for the complex linear span of the independent set $\left\{f_{1}^{\lambda}, \ldots, f_{d(\lambda)}^{\lambda}\right\}$. One may readily 
verify the formulas $f_{1}^{\lambda}\left(n_{0}^{-1}\right)=1 ; \lambda(a) v_{d(\lambda)}^{\lambda}=\Sigma \bar{f}_{i}^{\lambda}(a) v_{i}^{\lambda} ; \lambda^{*}(a) \phi_{d(\lambda)}^{\lambda}=$ $\Sigma f_{i}^{\lambda}(a) \phi_{i}^{\lambda} ; \Sigma f_{i}^{\lambda} \bar{f}_{i}^{\lambda}=1$ (the summations in these last three formulas are for $i=1, \ldots, d(\lambda))$.

For $\lambda$ in $\hat{G}$ we identify $\operatorname{End}\left(\mathrm{V}^{\lambda}\right)$ and $V^{\lambda} \otimes V^{\lambda^{*}}$ by corresponding $v \otimes \phi$ to the endomorphism sending $v^{\prime}$ to $\phi\left(v^{\prime}\right) v$; in particular $v_{i}^{\lambda} \otimes \phi_{j}^{\lambda}$ corresponds to $E^{i j, \lambda}$. Further equating $\operatorname{End}\left(V^{\lambda}\right)$ with $L^{2}(G)_{\lambda}=\mathscr{F}^{*}\left(\operatorname{End}\left(V^{\lambda}\right)\right)$ via the Fourier transform $\mathscr{F}$, we see that $L(a)$ corresponds to $\lambda(a) \otimes 1$ and $R(a)$ to $1 \otimes$ $\lambda^{*}(a)$, for $a$ in $G$.

2.5. Cyclic representations. A representation $\rho$ of $G$ is called cyclic if there is $v$ in $V^{\rho}$ so that $V^{\rho}$ equals the linear span of the orbit $\{\rho(a) v \mid a \in G\}$; a vector whose orbit spans $V^{\rho}$ is called a cyclic vector.

Proposition (2.5.1). (a) $\rho$ is cyclic if $\rho$ is in $\hat{G}$. (b) If $\rho_{1}$ and $\rho_{2}$ are in $\hat{G}$, then $\rho_{1} \otimes \rho_{2}$ is cyclic; in fact $v_{1} \otimes v_{2}$ is a cyclic vector if $v_{1}$ is a highest weight vector for $\rho_{1}$ and $v_{2}$ a lowest weight vector for $\rho_{2}$, and $v_{1} \neq 0, v_{2} \neq 0$.

Proof. (a) The linear span of an orbit is an invariant subspace. Thus any nonzero vector in $V^{\rho}$ is a cyclic vector for $\rho$ in $\hat{G}$.

(b) Let $v_{1}, v_{2}$ be as enunciated, set $v=v_{1} \otimes v_{2}$, and denote by $V$ the span of the orbit of $v$ under $\rho_{1} \otimes \rho_{2}$. We must show $V=V^{\rho_{1}} \otimes V^{\rho_{2}}$. Choose $u_{i} \in V^{\rho_{i}}, i=1$, 2. It suffices to show $u_{1} \otimes u_{2} \in v$. Now, $u_{1}$ is a linear combination of elements of the form $v_{1}, A v_{1}$, where $A=\rho_{1}\left(X_{1}\right) \ldots \rho_{1}\left(X_{r}\right) v_{1}$ $\left(\alpha(i) \in \Phi^{+}, X_{i} \in \mathcal{S}_{-\alpha(i)}\right)$. But

$$
\begin{aligned}
\left(\rho_{1} \otimes \rho_{2}\right)\left(X_{-\alpha}\right)\left(w_{1} \otimes v_{2}\right) & =\left(\rho_{1}\left(X_{-\alpha}\right) w_{1}\right) \otimes v_{2}+w_{1} \otimes \rho\left(X_{-\alpha}\right) v_{2} \\
& =\left(\rho_{1}\left(X_{-\alpha}\right) w_{1}\right) \otimes v_{2}
\end{aligned}
$$

for $\alpha \in \Phi^{+}, X_{-\alpha} \in \mathcal{G}_{-\alpha}, w_{1} \in V^{\rho_{1}}$. As $\left(\rho_{1} \otimes \rho_{2}\right)\left(\mathcal{G}_{C}\right) V \subset V$, one concludes $w_{1} \otimes v_{2} \in V$ for any $w_{1} \in V^{\rho_{1}}$. Now by (a) there are $c_{1}, \ldots, c_{r}$ in $\mathbf{C}$, $a_{1}, \ldots, a_{r}$ in $G$ with $u_{2}=\Sigma_{i} c_{i} \rho_{2}\left(a_{i}\right) v_{2}$; hence

$$
\begin{aligned}
u_{1} \otimes u_{2} & =\sum_{i} c_{i} u_{1} \otimes \rho_{2}\left(a_{i}\right) v_{2} \\
& =\sum_{i} c_{i}\left(\rho_{1} \otimes \rho_{2}\right)\left(a_{i}\right)\left(\rho_{1}\left(a_{i}^{-1}\right) u_{1} \otimes v_{2}\right) .
\end{aligned}
$$

With the previous remark, one concludes that $u_{1} \otimes u_{2} \in V$. Q.E.D.

3. The Borel-Weil Theorem and its consequences. We retain the notations of the preceding sections.

3.1. Borel-Weil Theorem. For $\mu$ in $\mathcal{T}_{\mathbf{C}}^{*}$ set

$$
\begin{aligned}
\Re(\mu) & =\left\{f \in C^{\infty}(G) \mid(R(X)+\mu(X)) f=0 \text { if } X \in \mathscr{T}_{\mathbf{C}}+\mathfrak{\Re}^{+}\right\}, \\
\mathfrak{R}^{\prime}(\mu) & =\left\{f \in C^{\infty}(G) \mid(L(X)+\mu(X)) f=0 \text { if } X \in \mathscr{T}_{\mathbf{C}}+\mathfrak{\Re}^{+}\right\} .
\end{aligned}
$$


Let $G_{\mu}$ denote the isotropy subgroup of $\mu$ in $G$ under the contragredient adjoint action, and let $\mathcal{G}_{\mu}$ denote the Lie algebra of $G_{\mu}$.

The implication of the usual Borel-Weil Theorem(see [12]) from that stated below is detailed in [7].

Theorem (3.1.1) (Borel-Weil Theorem). (a) $\Re(\mu)=0$ unless $-\mu$ is in $\Lambda^{+}$.

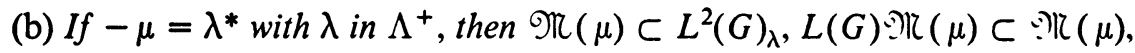
and the subrepresentation of $L$ in $\mathscr{R}(\mu)$ is equivalent to $\lambda$.

(c) For $\mu, \mu^{\prime}$ in $\mathcal{T}_{\mathbf{C}}^{*}, \mathfrak{T}(\mu) \cap \mathfrak{R}^{\prime}\left(\mu^{\prime}\right) \neq 0$ if and only if there is $\lambda$ in $\Lambda^{+}$with $-\mu=\lambda^{*},-\mu^{\prime}=\lambda$. If $\Re(\mu) \cap \Re^{\prime}\left(\mu^{\prime}\right)$ is not zero, then its dimension is 1 .

(d) $\Re(\mu)=\left\{f \in C^{\infty}(G) \mid(R(X)+\mu(X)) f=0\right.$ if $\left.X \in\left(\mathcal{G}_{\mu}\right)_{\mathrm{C}}+\Re^{+}\right\}$.

Proof. We assert that if $f$ is in $\mathscr{N}(\mu)$ then $f_{\gamma}=\mathscr{F}^{*}\left(\left(\mathscr{Y}(f)_{\gamma}\right)\right)$ is in $\mathbb{N}(\mu)$ for $\gamma$ in $\hat{G}$. Writing $g_{\gamma}=f-f_{\gamma}$ we have $f=f_{\gamma}+g_{\gamma}, f_{\gamma} \in L^{2}(G)_{\gamma}, g_{\gamma} \in$ $L^{2}(G)_{\gamma}^{\perp}$. Now as $R$ is unitary and preserves $L^{2}(G)_{\gamma}$, it also preserves $L^{2}(G)_{\gamma}^{\perp}$; hence the same is true of $R(X)+\mu(X)$ for any $X$ in $\mathcal{S}_{\mathbf{c}}$. Thus,

$$
(R(X)+\mu(X)) f_{\gamma}+(R(X)+\mu(X)) g_{\gamma}
$$

is the decomposition of $(R(X)+\mu(X)) f$ into orthogonal components in $L^{2}(G)_{\gamma}$ and $L^{2}(G)_{\gamma}^{\perp}$. In particular, if $(R(X)+\mu(X)) f=0$, then

$$
(R(X)+\mu(X)) f_{\gamma}=0=(R(X)+\mu(X)) G_{\gamma},
$$

from which the assertion follows.

We now show that for $f$ in $\Re(\mu)$ and $\lambda$ in $\Lambda^{+}, f=0$ unless $-\mu=\lambda^{*}$. In the identification of $L^{2}(G)_{\lambda}$ with $V^{\lambda} \otimes V^{\lambda^{*}}$, $\Re(\mu) \cap L^{2}(G)_{\lambda}$ corresponds to $V^{\lambda} \otimes\left\{\phi \in V^{\lambda^{*}} \mid\left(\rho_{\lambda^{*}}(X)+\mu(X)\right) \phi=0\right.$ if $\left.X \in \mathscr{T}_{\mathbf{C}}+\mathscr{\vartheta}^{+}\right\}$. But this latter set is zero unless $-\mu=\lambda^{*}$, by the uniqueness of highest weight of $V^{\lambda^{*}}$. This establishes (a). Now if $-\mu=\lambda^{*}$ with $\lambda$ in $\Lambda^{+}$, then we have that $\mathscr{R}(\mu) \subset$ $L^{2}(G)_{\lambda}, L(G) \Re(\mu) \subset \Re(\mu)$ (as $L$ and $R$ commute), and the subrepresentation of $L$ in $\mathscr{R}(\mu)$ is isomorphic to that of $\lambda \otimes 1$ in $V^{\lambda} \otimes V_{\lambda^{*}}^{\lambda^{*}}$. As $\operatorname{dim} V_{\lambda^{*}}^{\lambda^{*}}$ $=1$, the representation $\lambda \otimes 1$ of $G$ in $V^{\lambda} \otimes V_{\lambda^{*}}^{\lambda^{*}}$ is equivalent to that of $\lambda$. This establishes (b). Now if $\mu$ and $\mu^{\prime}$ are in $\mathscr{T}_{\mathbf{C}}^{*}$, we know by (a) that $\Re(\mu) \cap \Re^{\prime}\left(\mu^{\prime}\right)=0$ unless $\mu=-\lambda^{*}$ for some $\lambda$ in $\Lambda^{+}$. If $\mu=-\lambda^{*}$, with $\lambda$ in $\Lambda^{+}$, then, in the identification with $V^{\lambda} \otimes V^{\lambda^{*}}, \Re(\mu) \cap \Re^{\prime}\left(\mu^{\prime}\right)=\{v \in$ $V^{\lambda} \mid\left(\rho_{\lambda}(X)+\mu^{\prime}(X)\right) v=0$ if $\left.X \in \mathscr{T}_{\mathbf{C}}+\Re^{+}\right\} \otimes V_{\lambda^{*}}^{\lambda^{*}}$. The first factor is 0 unless $\mu^{\prime}=-\lambda$, by the uniqueness of highest weight in $V^{\lambda}$. If $-\mu^{\prime}=\lambda$, then $\Re(\mu) \cap \Re^{\prime}\left(\mu^{\prime}\right)$ corresponds to $V_{\lambda}^{\lambda} \otimes V_{\lambda^{*}}^{\lambda^{*}}$, which is a one-dimensional space. This proves (c). To prove (d), we need only show that $\rho_{\lambda^{*}}(X) V_{\lambda^{*}}^{\lambda^{*}} \subset$ $V_{\lambda^{*}}^{\lambda^{*}}$ if $X$ is in $\left(\mathcal{G}_{\mu}\right)_{\mathbf{C}}$ where $-\mu=\lambda^{*}$. But $\left(\mathcal{G}_{\mu}\right)_{\mathbf{C}}=\mathscr{T}_{\mathbf{C}}+\Sigma_{\alpha \in \Phi^{\prime}} \mathcal{G}_{\alpha}$, where $\Phi^{\prime}=\{\alpha \in \Phi \mid\{\alpha, \mu\}=0\}$. Now if $\alpha \in \Phi^{\prime} \cap \Phi^{+}$, then $V_{\lambda^{*}+\alpha}^{\lambda^{*}}=(0)$, as $\lambda^{*}$ is the highest weight. But then $V_{\lambda^{*}-\alpha}^{\lambda^{*}}=V_{\left.\sigma_{\alpha} \lambda^{*}+\alpha\right)}^{\lambda^{*}}=\rho_{\lambda^{*}}\left(n_{\alpha}\right) V_{\lambda^{*}+\alpha}^{\lambda^{*}}=(0)$, where 
$\sigma_{\alpha}=n_{\alpha} T$. Thus the highest weight space of $\lambda^{*}$ is invariant under $\left(\mathcal{G}_{\mu}\right)_{C}$. Q.E.D.

In view of the Borel-Weil Theorem, we introduce the notation $\mathscr{B}_{\lambda}=$ $\mathscr{R}\left(-\lambda^{*}\right), \mathscr{B}_{\lambda}^{0}=\mathscr{R}\left(-\lambda^{*}\right) \cap \mathscr{R}^{\prime}(-\lambda)$, for $\lambda$ in $\Lambda^{+}$. The subrepresentation of $L$ in $\mathscr{B}_{\lambda}$ is equivalent to $\lambda$, and $\mathscr{G}_{\lambda}^{0}$ is a one-dimensional subspace of $\mathscr{S}_{\lambda}$, in fact being the $\lambda$ weight-space of $L$ in $\mathscr{B}_{\lambda}$. We call $\mathscr{B}_{\lambda}$ the Borel-Weil realization of $\lambda$ in $\hat{G}=\Lambda^{+}$. Note that $\mathscr{B}_{\lambda}$ as here defined agrees with the notation introduced in 2.4 and that $f_{1}^{\lambda}$ is a spanning vector of $\mathscr{B}_{\lambda}^{0}$. We call $\cup_{\lambda \in \Lambda^{+}} \mathfrak{B}_{\lambda}^{0}$ the set of Borel-Weil functions.

THEOREM (3.1.2). $\mathscr{B}_{\lambda}^{0} \mathscr{B}_{\lambda^{\prime}}^{0}=\mathscr{B}_{\lambda+\lambda^{\prime}}^{0}$, for $\lambda, \lambda^{\prime}$ in $\Lambda^{+}$.

Proof. The Leibnitz rule for differentiating a product of functions shows immediately that $\mathfrak{B}_{\lambda}^{0} \mathscr{B}_{\lambda^{\prime}}^{0} \subset \mathfrak{B}_{\lambda+\lambda^{\prime}}^{0}$. Now, $\left(f_{1}^{\lambda} \cdot f_{1}^{\lambda^{\prime}}\right)\left(n_{0}^{-1}\right)=1$ (see 2.4). Thus $\mathscr{B}_{\lambda}^{0} \mathscr{B}_{\lambda^{\prime}}^{0} \neq 0$ and as $\mathscr{B}_{\lambda+\lambda^{\prime}}^{0}$ is one-dimensional, the result holds. Q.E.D.

Explicit formulas for Borel-Weil functions of the four classical series of compact simple Lie groups are detailed in [7].

3.2. Cartan product and tensor product. The results 3.2.1 and 3.2.4 below show how Borel-Weil realizations interact under point-wise multiplication and complex conjugation to yield realizations of the Cartan semigroup and tensor product operations.

THEOREM (3.2.1). $\mathscr{B}_{\lambda} \mathfrak{B}_{\nu}=\mathscr{B}_{\lambda+\nu}$.

Proof. The Leibnitz rule and the definition of the $\mathscr{G}_{\lambda}$ 's shows at once that $\mathscr{B}_{\lambda} \mathfrak{B}_{\nu} \subseteq \mathscr{B}_{\lambda+\nu} . \mathscr{P}_{\lambda} \mathscr{B}_{\nu}$ is invariant under $L$ and $\mathscr{B}_{\lambda+\nu}$ is irreducible under $L$. Thus either $\mathscr{B}_{\lambda} \mathscr{B}_{\nu}=(0)$ or $\mathscr{B}_{\lambda} \mathscr{B}_{\nu}=\mathscr{B}_{\lambda+\nu}$. But $0 \neq \mathscr{G}_{\lambda+\nu}^{0} \subseteq \mathfrak{G}_{\lambda} \mathscr{B}_{\nu}$, by (3.1.2). Thus, $\mathscr{B}_{\lambda} \mathscr{B}_{\nu}=\mathscr{B}_{\lambda+\nu}$. Q.E.D.

The next two results are preparatory to 3.2 .4 , but of interest in their own right.

THEOREM (3.2.2). The multiplication map $M: \mathscr{B}_{\lambda} \otimes \overline{\mathscr{B}}_{\nu} \rightarrow \mathscr{B}_{\lambda} \overline{\mathscr{B}}_{\nu}$ is a bijection, for $\lambda, \nu$ in $\Lambda^{+}$.

Proof. $M$ is clearly $C$-linear and surjective. To show $M$ is injective, choose $T$ with $M(T)=0$. We may write $T=\Sigma_{i j} T_{i j} f_{i}^{\lambda} \otimes \bar{f}_{j}^{\nu}$, where the $T_{i j}$ are certain numbers. Now set $\tau=\Sigma_{i, j} T_{i j} \tau_{i j}$, where $\left\{\tau_{i j}\right\}_{i, j}$ is the basis of $\left(V^{\lambda^{*}} \otimes V^{\nu}\right)^{*}$ dual to the basis $\left\{\phi_{i}^{\lambda} \otimes v_{j}^{\nu}\right\}_{i j}$ of $V^{\lambda^{*}} \otimes V^{\nu}$. The relations of 2.4 may be used to see that, for $a$ in $G$,

$$
\tau\left(\left(\lambda^{*} \otimes \nu\right)(a)\left(\phi_{d(\lambda)}^{\lambda} \otimes v_{d(\nu)}^{v}\right)\right)=M(T)(a) .
$$

Since $M(T)=0$, we conclude, with the assistance of 2.5.1(b), that $\tau=0$. But then $T_{i j=0}$ for all $i, j$ which shows $T=0$. Q.E.D. 
Proposition (3.2.3). For $\lambda$ in $\Lambda^{+}, R\left(n_{0}\right) \mathscr{B}_{\lambda^{*}}=\overline{\mathscr{G}}_{\lambda}$. Thus $R\left(n_{0}\right)$ provides a G-module equivalence between $\mathscr{B}_{\lambda^{*}}$ and $\overline{\mathscr{G}}_{\lambda}$.

Proof. Choose $f$ in $\mathscr{G}_{\lambda^{*}}$ and $X$ in $\mathscr{T}_{\mathbf{C}}+\mathfrak{T}^{+}$. Note that $\operatorname{Ad}\left(n_{0}^{-1}\right) X$ is also in ${\underline{T_{c}}}_{c} \Re^{+}$(here $\bar{X}=X_{1}-i X_{2}$ if $X=X_{1}+i X_{2}$, with $X_{1}, X_{2} \in \mathcal{G}$ ) and that $\overline{\lambda(\bar{X})}=-\lambda(X)($ as $\lambda(\mathcal{G}) \subset i \mathbf{R})$. Then,

$$
\begin{aligned}
R(X) R\left(n_{0}\right) \bar{f} & =R\left(n_{0}\right) \overline{R \overline{\left(\operatorname{Ad}\left(n_{0}^{-1}\right) X\right)} f}=R\left(n_{0}\right) \overline{\lambda *\left(\overline{\left.\operatorname{Ad}\left(n_{0}^{-1}\right) X\right) f}\right.} \\
& =\overline{\left(w_{0} \lambda^{*}\right)(\bar{X})} R\left(n_{0}\right) \bar{f}=-\overline{\lambda(\bar{X})} R\left(n_{0}\right) \bar{f}=\lambda(X) R\left(n_{0}\right) \bar{f} .
\end{aligned}
$$

Thus, $R\left(n_{0}\right) \bar{f}$ is in $\mathscr{B}_{\lambda}$, so $R\left(n_{0}\right) f$ is in $\overline{\mathscr{B}}_{\lambda}$. Q.E.D.

Corollary (3.2.4). The map $f \otimes g \rightarrow f . R\left(n_{0}\right) g$ yields an isomorphism of $G$-modules between $\mathscr{B}_{\lambda} \otimes \mathscr{B}_{\nu}$ and $\mathscr{B}_{\lambda} \overline{\mathscr{B}}_{\nu^{*}}$ for any $\lambda, \nu$ in $\Lambda^{+}$.

Of course, this isomorphism is nonunitary, in general; but still $\operatorname{ch}(\lambda \otimes \nu)=$ $\operatorname{ch}\left(\mathscr{B}_{\lambda} \overline{\mathscr{B}}_{\nu^{*}}\right)$.

4. $G$-module structure of certain infinite-dimensional representations. Throughout $\S 4, \lambda$ in $\Lambda^{+}$is fixed and for convenience usually assumed nonzero.

4.1. The Hopf bundle for $\lambda$. Set $K=G_{\lambda^{*}}=\left\{a \in G \mid \operatorname{ad}^{*}(a) \lambda^{*}=\lambda^{*}\right\}, M=$ $G / K, \mathcal{K}$ the Lie algebra of $K . K$ is a compact connected subgroup of $G$ (see 6.6.2 of [16]).

Proposition (4.1.1); There is a unique one-dimensional unitary representation $\chi: K \rightarrow S^{1}$ so that $\chi(\exp X)=e^{\lambda^{*}(X)}$ for $X \in \mathcal{K}$.

Proof. Since $K$ is compact connected, the given formula ensures the uniqueness of $\chi$. Since $\lambda^{*}(\mathscr{g}) \subset 2 \pi i \mathbf{Z}$ and $T$ is abelian, there does exist a character $\chi$ on $T$ so that $\chi(\exp H)=e^{\lambda^{*}(H)}$ for $H$ in $\mathcal{T}$. If $a T a^{-1}=T$ for $a$ in $K$, then the element $a$ represents an element of the Weyl group $W_{K}$ of $T$ in $K$; but $W_{K}$ is generated by the reflections $\sigma_{\alpha}$, where $\alpha$ is in $\Phi$ with $\mathcal{G}_{\alpha} \subset \mathscr{K}_{\mathbf{C}}$, and $\sigma_{\alpha} \lambda^{*}=\lambda^{*}$ for such $\alpha$; therefore $\chi\left(a t a^{-1}\right)=\chi(t)$. Thus, there is a unique continuous class function $\chi$ on $K$ extending $\chi$ on $T$ (see 4.32 of [1]). It is immediate that $\chi(\exp X)=e^{\lambda^{*}(X)}$ for $X$ in $\mathscr{K}$, and it remains to see that $\chi$ is a group homomorphism. For this we write $\mathscr{K}=\mathscr{Z}(\mathscr{K}) \oplus[\mathcal{K}, \mathscr{K}]$ and note that $\lambda^{*}([\mathscr{K}, \mathscr{K}])=0$, exp $\mathscr{Z}(\mathscr{K}) \subset Z(K)$, and the connected Lie subgroup with Lie algebra [ $\mathcal{K}, \mathcal{K}]$ is compact connected. Choose (see [15, p. 234]) $a, b$ in $K$, $X, Y$ in $\mathscr{K}$ with $\exp X=a, \exp Y=b$, and write $X=X_{1}+X_{2}, Y=Y_{1}+$ $Y_{2}$ according to the preceding decomposition of $\mathscr{K}$, and choose $Z_{2}$ in [ $\mathscr{K}, \mathscr{K}$ ] with $\exp X_{2} \exp Y_{2}=\exp Z_{2}$. Then one sees that $a b=\exp \left(X_{1}+Y_{1}+Z_{2}\right)$, from which it follows that $\chi(a b)=\chi(a) \chi(b)$. Thus $\chi$ is a continuous homomorphism from $K$ to $S^{1}$. It follows that $\chi$ is also smooth. Q.E.D. 
Set $K_{0}=\operatorname{ker} \chi ; K_{0}$ is a closed normal subgroup of $K$, and a closed subgroup of $G$. If $\lambda^{*}=0$, then $K_{0}=K=G$. Since $\lambda^{*} \neq 0$, the induced homomorphism $\tilde{\chi}: K / K_{0} \rightarrow S^{1}$ is a Lie group isomorphism, by means of which we identify $K / K_{0}$ and $S^{1}$. If $K=G$, then most of our theory is, while valid, trivial; as examples $\lambda^{*}=w_{1}+\cdots+w_{n}, G=U(n), \chi=\operatorname{det}$; and $\chi=\lambda^{*}$ if $G$ is abelian.

Set $P=G / K_{0}$ and let $\pi: P \rightarrow M$ send $a K_{0}$ to $a K$. Using $\chi$, we may define $P \times S^{1} \rightarrow P$ by $\left(a K_{0}, \zeta\right) \rightarrow(a x) K_{0}$, where $x \in K$ is chosen so that $\chi(x)=\zeta$. Then $\pi$ is the projection for a principal $S^{\prime}$ bundle, which we refer to as the Hopf bundle for $\lambda$. We show first how to describe this bundle in terms of the element $\lambda^{*}$ of $\hat{G}$.

Proposition (4.1.2). The map from $G$ to $V^{\lambda^{*}}$ sending a to $\lambda^{*}(a) \phi_{d_{\lambda}}^{\lambda}$ determines an $S^{1}$-bundle equivalence of $\pi$ with $P_{0} \rightarrow^{\pi_{0}} M_{0}$, where $P_{0}$ is the orbit of $\phi_{d_{\lambda}}^{\lambda}$ in $V^{\lambda^{*}}, M_{0}$ is the orbit of $V_{\lambda^{*}}^{\lambda^{*}}$ in the projective representation determined by $\lambda^{*}$, and $\pi_{0}$ is the restriction to $P_{0}$ of the natural projection. The $S^{1}$ action on $P_{0}$ is induced by scalar multiplication in $V^{\lambda^{*}}$.

Proof. Several steps are required in the proof. Abbreviate $\phi_{d_{\lambda}}^{\lambda}$ by $\phi, V^{\lambda^{*}}$ by $V$.

Step 1. $K=\left\{a \in G \mid \lambda^{*}(a) V_{\lambda^{*}}=V_{\lambda^{*}}\right\}$.

Proof OF STEP 1. Set $K^{\prime}=\left\{a \in G \mid \lambda^{*}(a) V_{\lambda^{*}}=V_{\lambda^{*}}\right\}$, $K^{\prime}$ the Lie algebra of $K^{\prime}$. There is $\chi^{\prime}$ in $\hat{K}^{\prime}$, defined by $\lambda^{*}(a) \phi=\chi^{\prime}(a) \phi, a \in K^{\prime}$. Let $\theta \in \mathcal{K}_{\mathbf{C}}^{\prime *}$ be the infinitesimal representation for $\chi^{\prime}$.

The proof of $3.1 .1(\mathrm{~d})$ establishes that $\mathscr{H}^{\circ} \subset \mathcal{K}^{\prime}$. Since $K^{*}$ is connected, $\mathscr{K} \subset \mathscr{K}^{\prime}$.

We claim $\theta$ is the restriction to $\mathcal{K}_{\mathbf{C}}^{\prime}$ of $\lambda^{*}$ in $\mathcal{G}_{\mathbf{C}}^{*}$. Clearly $\lambda^{*}$ and $\theta$ agree on $\mathcal{T}_{\mathbf{c}}$. Now $\mathcal{K}_{\mathbf{C}}^{\prime}$ is the sum of $\mathcal{T}_{\mathbf{c}}$ and the span of certain root vectors; this latter span being contained in the derived algebra [ $\mathcal{H}_{\mathbf{c}}^{\prime}, \mathcal{K}_{\mathbf{c}}^{\prime}$ ] of $\mathcal{K}_{\mathbf{C}}^{\prime}, \boldsymbol{\theta}$ vanishes on it, as does $\lambda^{*}$. Thus $\lambda^{*}$ agrees with $\theta$ on $\mathcal{K}_{\mathbf{C}^{*}}^{\prime}$

Next we show $K^{\prime} \subset K$. Fix $X$ in $\mathscr{K}^{\prime}, a$ in $K^{\prime}$. Then

$$
\begin{aligned}
\lambda^{*}(\operatorname{ad}(a) X) \cdot \phi & =\theta(\operatorname{ad}(a) X) \cdot \phi=\rho_{\lambda^{*}}(\operatorname{ad}(a) X) \cdot \phi \\
& =\rho_{\lambda^{*}}(a) \rho_{\lambda^{*}}(X) \rho_{\lambda^{*}}\left(a^{-1}\right) \cdot \phi=\chi^{\prime}(a) \theta(X) \chi^{\prime}\left(a^{-1}\right) \cdot \phi \\
& =\theta(X) \cdot \phi=\lambda^{*}(X) \cdot \phi .
\end{aligned}
$$

Thus, $\left.\left(\operatorname{ad}^{*}(a) \lambda^{*}\right)\right|_{\mathcal{K}^{\prime}}=\left.\lambda^{*}\right|_{\mathscr{K}^{\prime}}$. Since $\operatorname{ad}(a) \mathcal{K}^{\prime} \subset \mathcal{K}^{\prime}$, also $\operatorname{ad}(a) \mathcal{K}^{\prime \perp} \subset \mathfrak{K}^{\prime \perp}$; and one has $\left.\left(\operatorname{ad}^{*}(a) \lambda^{*}\right)\right|_{\mathcal{K}^{\prime \perp}}=0=\left.\lambda^{*}\right|_{\mathscr{K}^{\prime \perp}}$. We conclude that $\operatorname{ad}^{*}(a) \lambda^{*}=\lambda^{*}$, i.e., $a$ is in $K$. This concludes Step 1 .

Step 2. $K_{0}=\left\{a \in G \mid \lambda^{*}(a) \cdot \phi=\phi\right\}$.

This is trivial when Step 1 is used. 
Step 3. Conclusion of proof of proposition. We may, by Steps 1 and 2 , define $\tilde{F}: P \rightarrow P_{0}$ and $F: M \rightarrow M_{0}$ by $\tilde{F}\left(a K_{0}\right)=\lambda^{*}(a) \phi, F(a K)=\lambda^{*}(a) V_{\lambda^{*}}^{\lambda^{*}}$, and moreover $\tilde{F}$ and $F$ are bijections. $\tilde{F}$ is easily seen to be an equivalence of $S^{1}$ bundles, with $\pi_{0} \tilde{F}=F \pi$. Q.E.D.

4.2. Main theorem. Recall from $\S 1$ the subrepreseniations $\Gamma$ and $\Gamma_{k}(k \in \mathbf{Z})$ of the left-regular representation of $G$.

We relate our discussion here to that of $\$ 3$ by introducing

$$
\Gamma_{p, q}=\mathscr{B}_{p \lambda} \overline{\mathscr{G}}_{q \lambda}, \quad p, q \in \mathbf{Z}^{+} .
$$

Proposition (4.2.1). (a) $\Gamma_{k} \subseteq \Gamma$ for $k$ in $\mathbf{Z}$.

(b) $\Gamma_{p, q} \subseteq \Gamma_{p-q}$ for $p, q$ in $\mathbf{Z}^{+}$.

Proof. (a) is clear. For (b), choose $f \in \mathscr{Q}_{p \lambda}, g \in \mathscr{Q}^{\prime}{ }_{q \lambda}$, and $x \in K$. Then

$$
\begin{aligned}
R(x)(f \bar{g}) & =(R(x) f)(R(x) \bar{g})=(R(x) f) \overline{(R(x) g)} \\
& =\chi(x)^{p} \overline{\chi(x)^{q}} f \bar{g}=\chi(x)^{p-q}(f \bar{g}) .
\end{aligned}
$$

Thus $f \bar{g} \in \Gamma_{p-q^{\prime}}$ as desired. Q.E.D.

Proposition (4.2.2). The algebraic sum $\Sigma_{p . q \in \mathbf{Z}^{+}} \Gamma_{p . q}$ is dense in $\Gamma$.

Proof. The continuous functions $\Gamma^{\prime}$ in $\Gamma$ are dense in $\Gamma . \Gamma^{\prime}$ may be regarded as $C(P)$, the continuous functions on $P$ (see (4.1.2)). It will suffice to show $\tilde{\Gamma}=\Sigma_{p, q} \Gamma_{p, q}$ is dense in $\Gamma^{\prime}$ with respect to the sup-norm. As $P$ is compact, this density will follow from the Stone-Weierstrass Theorem, provided we show that $\tilde{\Gamma}$ has the following properties:

(i) If $f, g \in \tilde{\Gamma}$, and $z \in \mathbf{C}$ then $f_{\tilde{\tau}}+z g, f g$, and $\bar{f} \in \tilde{\Gamma}$.

(ii) For all $u \in P$, there is $f$ in $\tilde{\Gamma}$ with $f(u) \neq 0$.

(iii) For all $u_{1}, u_{2}$ in $P$ with $u_{1} \neq u_{2}$, there is $f$ in $\tilde{\Gamma}$ with $f\left(u_{1}\right) \neq f\left(u_{2}\right)$.

We show $\tilde{\Gamma}$ has these three properties.

ProQf OF (i). $\Gamma_{p, q}$ is a complex subspace and $\bar{\Gamma}_{p, q}=\Gamma_{q, p}$, so $f+z g$ and $\bar{f}$ are in $\Gamma$. From (3.6),

$$
\Gamma_{p, q} \Gamma_{p^{\prime}, q^{\prime}}=\mathscr{B}_{p \lambda} \overline{\mathscr{G}}_{q \lambda} \mathscr{B}_{p^{\prime} \lambda} \overline{\mathscr{B}}_{q^{\prime} \lambda}=\mathscr{G}_{\left(p+p^{\prime}\right) \lambda} \overline{\mathscr{G}}_{\left(q+q^{\prime}\right) \lambda}=\Gamma_{p+p^{\prime}, q+q^{\prime}}
$$

Thus $f g$ is in $\tilde{\Gamma}$.

ProOF OF (ii). We will in fact find the desired functions for (ii) and (iii) in $\Gamma_{1,0}=\mathscr{B}_{\lambda}$. As $\mathscr{B}_{\lambda} \neq 0$, choose $f_{0}$ in $\mathscr{G}_{\lambda}, a_{0}$ in $G$ with $f_{0}\left(a_{0}\right) \neq 0$. Then for $a$ in $G, 0 \neq f_{0}\left(a_{0}\right)=f_{0}\left(a_{0} a^{-1} a\right)=\left(L_{a a_{0}-1} f_{0}\right)(a)$. But $L_{a a_{0}-1} f_{0}$ is in $o_{\lambda}$. This proves (ii).

Proof of (iii). As in (ii), the homogeneity of $P$ and the result $L(G) \mathscr{M I}_{\lambda} \subset$ $\mathscr{B}_{\lambda}$ reduces the question to demonstrating the validity of the following statement:

$$
\forall a \notin K_{0} \quad \exists f \in \stackrel{Q B}{\lambda} \quad f(a) \neq f(e)
$$


Now the statement in question is false if and only if

$$
\exists a \notin K_{0} \quad \forall f \in \mathscr{B}_{\lambda} \quad f(a)=f(e) .
$$

Thus, we need to show that

$$
K_{0} \supset\left\{a \in G \mid \forall f \in \mathscr{B}_{\lambda}, \quad f(a)=f(e)\right\} .
$$

Let $S$ be the set we want $K_{0}$ to contain. Then, using again $L(G)\left(\mathscr{T}_{\lambda} \subset \mathscr{G}_{\lambda}\right.$, one shows

$$
S=\left\{a \in G \mid \forall f \in \mathscr{G}_{\lambda}, \quad R(a) f=f\right\} .
$$

Letting $\mathscr{B}_{\lambda}^{\prime}=\mathscr{F}\left(\mathscr{B}_{\lambda}\right) \subset V^{\lambda} \otimes V^{\lambda^{*}}$, we recall that $\mathscr{G}_{\lambda}^{\prime}=V^{\lambda} \otimes\{\phi\}\left(\phi=\phi_{d_{\lambda}}^{\lambda}\right)$ so $S=\left\{a \in G \mid \lambda^{*}(a) \cdot \phi=\phi\right\}$. Thus $S=K_{0}$ by Step 2 of the proof of (4.1.2). Q.E.D.

Proposition (4.2.3). $\Gamma=\bigoplus_{k \in \mathbf{z}} \Gamma_{k}$, a Hilbert space direct sum.

Proof. Choose distinct integers $k$ and $l$; since $\lambda^{*} \neq 0$, we may choose $x$ in $K$ with $\chi(x)^{k-l} \neq 1$. Then for $f$ in $\Gamma_{k}$ and $g$ in $\Gamma_{1},\{f, g\}=\chi(x)^{k-1}\{f, g\}=$ 0 ; thus $\Gamma_{k} \perp \Gamma_{l}$. By the previous two propositions $\Sigma_{k \in \mathbf{Z}} \Gamma_{k}$ is dense in $\Gamma$; (4.2.3) follows. Q.E.D.

Proposition (4.2.4). $\Gamma_{p, q} \subset \Gamma_{p+1, q+1}$, for $p, q$ in $\mathbf{Z}^{+}$.

Proof.

$$
\Gamma_{p+1, q+1}=\mathscr{B}_{(p+1) \lambda} \overline{\mathscr{B}}_{(q+1) \lambda}=\mathscr{G}_{p \lambda} \overline{\mathscr{G}}_{q \lambda} \mathscr{G}_{\lambda} \overline{\mathscr{B}}_{\lambda} .
$$

Thus it suffices to show $1 \in \mathscr{B}_{\lambda} \overline{\mathscr{G}}_{\lambda}$. But from 2.4, $1=\Sigma_{i} f_{i} \hat{f}_{i}^{\lambda} \in \mathscr{G}_{\lambda} \overline{\mathscr{G}}_{\lambda}$. Q.E.D.

The same proof shows that $\mathscr{B}_{\nu_{1}} \overline{\mathscr{G}}_{\nu_{2}} \subseteq \mathscr{G}_{\nu_{1}+\nu_{3}} \overline{\mathscr{B}}_{\nu_{1}+\nu_{3}}$, for any $\nu_{1}, \nu_{2}, \nu_{3}$ in $\Lambda^{+}$.

Proposition (4.2.5). Let $\nu_{1}, \nu_{2}$ be in $\Lambda^{+}$. Then

$$
\left\{\operatorname{ch}\left(\nu_{1}+n \nu_{2} \otimes n \nu_{2}^{*}\right)\right\}_{n=0}^{\infty}, \quad\left\{\operatorname{ch}\left(n \nu_{2} \otimes \nu_{1}+n \nu_{2}^{*}\right)\right\}_{n=0}^{\infty}
$$

are increasing sequences in $\mathcal{S}$, bounded above by $d$. Thus, their limits exist in $\$$.

Proof. By (3.1.1), (3.2.4) and (4.2.4),

$$
\begin{aligned}
& \operatorname{ch}\left(\left(\nu_{1}+n \nu_{2}\right) \otimes n \nu_{2}^{*}\right)=\operatorname{ch}\left(\mathscr{B}_{\nu_{1}+n \nu_{2}} \otimes \mathscr{B}_{n \nu_{2}^{*}}\right)=\operatorname{ch}\left(\mathscr{B}_{\nu_{1}} \mathscr{B}_{n \nu_{2}} \overline{\mathscr{P}}_{n \nu_{2}}\right) \\
& \leqslant \operatorname{ch}\left(\mathscr{G}_{\nu_{1}} \mathscr{B}_{n \nu_{2}} \overline{\mathscr{B}}_{n \nu_{2}} \mathscr{G}_{\nu_{2}} \overline{\mathscr{G}}_{\nu_{2}}\right)=\operatorname{ch}\left(\nu_{1}+(n+1) \nu_{2} \otimes(n+1) \nu_{2}^{*}\right),
\end{aligned}
$$

so the first sequence is increasing. Since $\operatorname{ch}\left(\mathscr{B}_{\mu_{1}} \overline{\mathscr{B}}_{\mu_{2}}\right) \leqslant d$ for $\mu_{1}, \mu_{2}$ in $\Lambda^{+}$, the above expression assures the first sequence bounded above by $d$. This proves the proposition for the first sequence; the proof for the second sequence is entirely analogous. Q.E.D. 
THEOREM (4.2.6).

$\operatorname{ch}\left(\Gamma_{k}\right)=\lim _{n \rightarrow \infty} \operatorname{ch}\left(k \lambda+n \lambda \otimes n \lambda^{*}\right), \quad \operatorname{ch}\left(\Gamma_{-k}\right)=\lim _{n \rightarrow \infty} \operatorname{ch}\left(n \lambda \otimes k \lambda^{*}+n \lambda^{*}\right)$ for $k$ in $\mathbf{Z}^{+}$. The sequences in $\mathcal{S}$ involved are increasing and bounded above.

Proof. By (4.2.5) we know the limits involved exist. The argument being similar in both cases, consider $\Gamma_{k}$; call the limit in question $f$. From 3.2.4, $\operatorname{ch}\left(\Gamma_{k+n, n}\right)=\operatorname{ch}\left(k \lambda+n \lambda \otimes n \lambda^{*}\right)$; now application of (4.2.1)-(4.2.4) shows that $f=\operatorname{ch}\left(\Gamma_{k}\right)$. Q.E.D.

4.3. Some formulas holding for $\Gamma_{k}$ in general. Let $\lambda \neq 0$ in $\Lambda^{+}$be chosen. For $f$ in $\mathcal{S}$, define $f^{*}$ in $\mathcal{S}$ by $f^{*}(\mu)=f\left(\mu^{*}\right)$, for $\mu$ in $\Lambda^{+}$.

Proposition (4.3.1). For $k$ in $\mathbf{Z}^{+}$,

(a) $\operatorname{ch}\left(\Gamma_{k}(\lambda)\right) \geqslant \sum_{n=0}^{\infty} \operatorname{ch}\left(k \lambda+n \lambda+n \lambda^{*}\right)$,

(b) $\operatorname{ch}\left(\Gamma_{-k}(\lambda)\right)=\operatorname{ch}\left(\Gamma_{k}(\lambda)\right)^{*}=\operatorname{ch}\left(\Gamma_{k}\left(\lambda^{*}\right)\right)$,

(c) $\operatorname{ch}\left(\Gamma_{k}(\lambda)\right)=\operatorname{ch}\left(\Gamma_{1}(k \lambda)\right)$, if $k \neq 0$.

PRoof. (a) $\operatorname{ch}\left(\Gamma_{k}\right)=\lim _{n \rightarrow \infty} \operatorname{ch}\left(k \lambda+n \lambda \otimes n \lambda^{*}\right) \geqslant \operatorname{ch}\left(k \lambda+N \lambda \otimes N \lambda^{*}\right)$, for fixed $N \in \mathbf{Z}^{+}$. But $\operatorname{ch}\left(k \lambda+N \lambda \otimes N \lambda^{*}\right) \geqslant \operatorname{ch}\left(k \lambda+N \lambda+N \lambda^{*}\right)$, by the usual realization of the Cartan product [11, p. 111]. (a) follows.

$$
\begin{aligned}
\operatorname{ch}\left(\Gamma_{k}(\lambda)\right)^{*} & =\left(\lim _{n \rightarrow \infty} \operatorname{ch}\left(k \lambda+n \lambda \otimes n \lambda^{*}\right)\right)^{*} \\
& =\lim _{n \rightarrow \infty} \operatorname{ch}\left(n \lambda \otimes k \lambda^{*}+n \lambda^{*}\right)=\operatorname{ch}\left(\Gamma_{-k}(\lambda)\right) .
\end{aligned}
$$

Similarly $\operatorname{ch}\left(\Gamma_{k}\left(\lambda^{*}\right)\right)=\lim _{n \rightarrow \infty} \operatorname{ch}\left(k \lambda^{*}+n \lambda^{*} \otimes n \lambda\right)=\operatorname{ch}\left(\Gamma_{-k}(\lambda)\right)$.

$$
\begin{aligned}
\Gamma_{k}(\lambda) & =\left\{f \in L^{2}(G) \mid R(\exp X) f=\left(e^{\lambda^{*}(X)}\right)^{k} f, X \in \mathcal{G}_{\lambda}\right\} \\
& =\left\{f \in L^{2}(G) \mid R(\exp X) f=e^{k \lambda^{*}(X)} f, X \in \mathcal{G}_{\lambda}\right\} \\
& =\Gamma_{1}(k \lambda), \quad \text { as } \mathcal{G}_{\lambda}=\mathcal{G}_{k \lambda} \text { since } \lambda \neq 0 \text {. Q.E.D. }
\end{aligned}
$$

One might be better able to think about the infinite series in part (a) of the proposition if it were summed in a closed form. This idea may be formalized as follows. In order to avoid an additional symbol, let $\mathcal{S}$ now represent the set of all Z $\mathbf{Z}$-valued functions defined on $\Lambda^{+}$(earlier, we restricted to $\mathbf{Z}^{+}$-valued functions). The set $\left\{\operatorname{ch}(\lambda) \mid \lambda \in \Lambda^{+}\right\}$is a $Z$-independent subset and we may write $f=\Sigma_{\lambda \in \Lambda^{+}} f(\lambda) \operatorname{ch}(\lambda)$ for $f$ in $\mathcal{S}$. Define $\operatorname{ch}(\lambda) \operatorname{ch}\left(\lambda^{\prime}\right)=\operatorname{ch}\left(\lambda+\lambda^{\prime}\right)$, and extend this definition to elements $f, g$ in $\S$ by

$$
f g=\sum_{\lambda, \lambda^{\prime}} f(\lambda) g\left(\lambda^{\prime}\right) \operatorname{ch}\left(\lambda+\lambda^{\prime}\right)
$$

whenever the summation converges absolutely to an element of $\S$. In particular $f g$ is defined if either $f$ or $g$ is 0 off some finite set. Notice that $\operatorname{ch}(0) f=f$ for $f$ in $\mathcal{S}$. By introducing the formal identity $1 /(1-x)=$ $\sum_{n=0}^{\infty} x^{n}$, for elements $x$ in $\mathcal{S}$ whose powers are defined, we obtain the expression 


$$
\sum_{n=0}^{\infty} \operatorname{ch}\left(k \lambda+n \lambda+n \lambda^{*}\right)=\frac{\operatorname{ch}(k \lambda)}{\operatorname{ch}(0)-\operatorname{ch}\left(\lambda+\lambda^{*}\right)} .
$$

We will use such formalism without comment in the sequel.

4.4. The usual representation of $S U(n+1), n \geqslant 1$. Investigation of the present example was suggested to the author J. W. Robbin and led to the main theorem when correctly viewed. Namely, we consider $G=S U(n+1)$, $n \geqslant 1$, in its natural representation on $\mathbf{C}^{n+1}$. If $\left\{e_{i}\right\}_{i=1, \ldots, n+1}$ is the usual basis of $\mathrm{C}^{n+1}$, then $a e_{i}=\sum_{j} a_{j i} e_{j}$, for $a \in G$. The highest weight of this representation is $\Lambda_{1}$ with weight vector $e_{1}$. In view of (4.1.1) we should take $\lambda^{*}=\Lambda_{1}, \lambda=\Lambda_{n}, e_{1}=\phi=\phi_{d_{\lambda}}^{\lambda}$. The basis $\left\{f_{k}^{\lambda}\right\}_{i=1, \ldots, n+1}$ of $\mathrm{GB}_{\lambda}$ (see proof of (3.2.2)) may be regarded as the restrictions $\left\{z_{i}\right\}_{i=1, \ldots, n+1}$ to $G e_{1}=S^{2 n+1}=$ $P_{0}$ of the complex coordinate functions on $\mathbf{C}^{n+i}$. Employing the usual multinomial notation $Z^{I} \bar{Z}^{J}=Z_{1}^{I_{1}} \ldots \bar{Z}_{n+1}^{J_{n+1}}$, we see that $\Gamma_{p, q}=\mathscr{G}_{p \lambda} \overline{\mathscr{G}}_{q \lambda}=$ $\mathscr{G}_{\lambda} p \overline{\mathscr{G S}_{\lambda}^{q}}$ is spanned by $\left\{Z^{I} \bar{Z}^{J}|| I\left|=I_{1}+\cdots+I_{n+1}=p,\right| J \mid=J_{1}+\cdots+\right.$ $\left.J_{n+1}=q\right\}$. The inclusions $\Gamma_{p, q} \subset \Gamma_{p+1, q+1}$ of (4.2.4) result from the fact that $\sum_{i=1}^{n+1} z_{i} \bar{z}_{i}=1$ on $P_{0} . M_{0}$ is $\mathbf{C} P^{n}$ and $\pi_{0}: P_{0} \rightarrow M_{0}$ is the (usual) Hopf map.

We see that $\mathscr{B}_{\lambda}$ may be regarded as the restriction to $P_{0}$ of the linear functions on $\mathbf{C}^{n+1}$. Thus, $\mathscr{B}_{\lambda}$ may be regarded as sections of the line bundle dual to the Hopf bundle; this dual Hopf bundle is associated with the principal $S^{1}$ bundle $\pi_{0}^{*}: P_{0}^{*} \rightarrow M_{0}$, where $P_{0}^{*}=P_{0}, \pi_{0}^{*}=\pi_{0}$, but $\psi \cdot \zeta=\zeta^{-1}$. $\psi$ for $\psi \in P_{0}^{*}, \zeta \in S^{1}$.

Proposition (4.4.1).

$$
\begin{aligned}
\operatorname{ch}\left(\Gamma_{k}\right) & =\frac{\operatorname{ch}(\lambda)^{k}}{\operatorname{ch}(0)-\operatorname{ch}\left(\lambda+\lambda^{*}\right)}, \quad k \in \mathbf{Z}^{+} . \\
\operatorname{ch}(\Gamma) & =\frac{\operatorname{ch}(0)}{(\operatorname{ch}(0)-\operatorname{ch}(\lambda))\left(\operatorname{ch}(0)-\operatorname{ch}\left(\lambda^{*}\right)\right)} .
\end{aligned}
$$

Proof. (a) The point here is that

$$
\operatorname{ch}\left((p+1) \lambda+(q+1) \lambda^{*}\right)=\operatorname{ch}\left(\Gamma_{p+1 . q+1}\right)-\operatorname{ch}\left(\Gamma_{p . q}\right)
$$

this formula is proved using the Weyl dimension formula. The result then follows from (4.3).

(b) $\operatorname{ch}(\Gamma)=\sum_{k=-\infty}^{\infty} \operatorname{ch}\left(\Gamma_{k}\right)$. Setting $x=\operatorname{ch}(\lambda), y=\operatorname{ch}\left(\lambda^{*}\right)$ and using (a) and (4.3), one has (setting $\operatorname{ch}(0)=1$ )

$$
\begin{aligned}
\operatorname{ch}(\Gamma) & =\left[(1-x)^{-1}+(1-y)^{-1}-1\right](1-x y)^{-1} \\
& =(1-x)^{-1}(1-y)^{-1} \text {. Q.E.D. }
\end{aligned}
$$

4.5. Use of Steinberg's formula. The expression for $\operatorname{ch}\left(\Gamma_{k}\right)$ may be said to completely solve the question of $\Gamma_{k}$ 's $G$-module structure, as it expresses 
$\operatorname{ch}\left(\Gamma_{k}\right)$ in terms of certain $\operatorname{ch}\left(\nu_{1} \otimes \nu_{2}\right), \nu_{1}, \nu_{2}$ in $\hat{G}$. There is a certain closed expression for a general 'outer' multiplicity $\operatorname{ch}\left(\nu_{1} \otimes \nu_{2}\right)\left(\nu_{3}\right), \nu_{1}, \nu_{2}, \nu_{3} \in \Lambda^{+}$, namely Steinberg's formula $[8$, p. 141].

Steinberg's formula has certain drawbacks as a computational device, requiring as it does a double summation over the Weyl group and a knowledge of Kostant's partition function. The interested reader may refer to the references in [3, p. 120] for examples.

One question arising in the computation of

$$
\operatorname{ch}\left(\Gamma_{1}(\lambda)\right)(\nu)=\lim _{n \rightarrow \infty} \operatorname{ch}\left(\lambda+n \lambda \otimes n \lambda^{*}\right)(\nu)
$$

is the determination of the lowest value $n(\lambda, \nu)$ of $n$ at which

$$
\operatorname{ch}\left(k \lambda+n \lambda \otimes n \lambda^{*}\right)(\nu)=\operatorname{ch}\left(\Gamma_{1}(\lambda)\right)(\nu) .
$$

At present the author has no general information regarding this function $n(\lambda, \nu)$.

4.6. Frobenius reciprocity; $S U(2)$. Our representation $\Gamma_{k}$ is an induced representation. Namely, it is the unitary representation of $G$ induced from the unitary representation $\chi^{-k}$ of the closed subgroup $K=G_{\lambda^{*}}, \chi$ being the character for $\lambda^{*}$ on $K$. As with all unitarily induced representations of $G$, we may analyze $\Gamma_{k}$ by means of the Frobenius reciprocity relation. When used in coordination with such formulas as those of Kostant and Freudenthal [8, pp. $122,138]$, the following Frobenius reciprocity statement is very useful computationally for regular $\lambda$.

Proposition (4.6.1). $\operatorname{ch}\left(\Gamma_{k}(\lambda)\right)(\nu)=\operatorname{dim}\left\{v \in V^{v} \mid \nu(x) v=\chi^{-k}(x) v\right.$ if $x \in$ $K\}$. In particular, $\operatorname{ch}\left(\Gamma_{k}(\lambda)\right)(\nu) \leqslant \operatorname{dim} V_{k \lambda}^{\nu}$, with equality occurring if $\lambda$ is regular.

PROOF. $\operatorname{ch}\left(\Gamma_{k}\right)(\nu)=\operatorname{dim} \operatorname{Hom}_{G}\left(V^{\nu}, \Gamma_{k}\right)$, so by 5.3 .6 of [16], $\operatorname{ch}\left(\Gamma_{k}\right)(\nu)=$ $\operatorname{dim} \operatorname{Hom}_{K}\left(V^{\nu}, \chi^{-k}\right)$, where $\operatorname{Hom}_{K}\left(V^{\nu}, \chi^{-k}\right)=\left\{\psi \in V^{\nu^{*}} \mid \psi(\nu(x) v)=\right.$

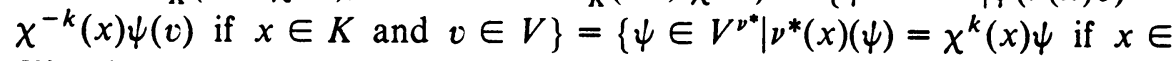
$K$ \}. Along with (4.3) one concludes $\operatorname{ch}\left(\Gamma_{k}\right)(\nu)=\operatorname{ch}\left(\Gamma_{k}\right)^{*}\left(\nu^{*}\right)=\operatorname{ch}\left(\Gamma_{-k}\right)\left(\nu^{*}\right)=$ $\operatorname{dim}\left\{v \in V^{v} \mid v(x)(v)=\chi^{-k}(x) v\right.$ if $\left.x \in K\right\}$. This shows the first formula of the proposition. Now since $\chi^{-k}(\exp H)=e^{-k \lambda^{*}(H)}$ for $H$ in $T$, the set whose dimension equals $\operatorname{ch}\left(\Gamma_{k}(\lambda)\right)(\nu)$ is contained in $V_{-\lambda^{*}}^{v}$, with the containment being equality when $\lambda$ is regular. Applying (2.3), one gets $\operatorname{ch}\left(\Gamma_{k}(\lambda)\right)(\nu) \leqslant$ $\operatorname{dim} V_{-k \lambda^{*}}^{v}=\operatorname{dim} V_{w_{0}(k \lambda)}^{\nu}=\operatorname{dim} V_{k \lambda}^{\nu}$, with equality if $\lambda$ is regular. Q.E.D.

The proposition yields $\operatorname{ch}\left(\Lambda_{k}\right)$ at once for $G=S U(2)$. Namely, suppose $\lambda=m \Lambda_{1}, m \in \mathbf{Z}^{+}, m \neq 0$. Suppose $\nu=n \Lambda_{1}, n \in \mathbf{Z}^{+}$. Then $\operatorname{dim} V_{k \lambda}^{v}=$ $\operatorname{dim} V_{k m \Lambda_{1}}^{n \Lambda}=1$ if $-n \leqslant k m \leqslant n$ and $k m \equiv n(\bmod 2), 0$ otherwise. Thus, if $x=\operatorname{ch}\left(\Lambda_{1}\right)$,

$$
\operatorname{ch}\left(\Lambda_{k}\right)=\sum_{n=0}^{\infty} \operatorname{ch}\left((k m+2 n) \Lambda_{1}\right)=\frac{x^{k m}}{\operatorname{ch}(0)-x^{2}} .
$$


When $m=1$, we recover a formula of (4.3) as here $\lambda=\lambda^{*}$.

4.7. $S U(3)$. We introduce notation which is convenient for the algebraic expressions for $\operatorname{ch}\left(\Lambda_{1}(\lambda)\right), \operatorname{ch}\left(\Gamma_{0}(\lambda)\right), \lambda \neq 0 \in \Lambda^{+}$. Recalling the usual weightroot notations for $S U$ (3) (see [8]), set $\alpha=2 \Lambda_{1}-\Lambda_{2}, \beta=-\Lambda_{1}+2 \Lambda_{2}, \delta=\alpha$ $+\beta=\Lambda_{1}+\Lambda_{2}$; where $\Lambda_{1}, \Lambda_{2}$ are usual fundamental weights; $\Phi^{+}=\{\alpha, \beta$, $\delta$ \}. In $\mathcal{S}$ set $x_{i}=\operatorname{ch}\left(\Lambda_{i}\right), i=1,2, A=x_{1}^{2} X_{2}^{-1}, B=x_{1}^{-1} x_{2}^{2} ; \operatorname{ch}(0)=1$ in $\delta$.

Proposition (4.7.1). Let $\lambda=m_{1} \Lambda_{1}+m_{2} \Lambda_{2} \neq 0 \in \Lambda^{+}$. Then:

(a) If $m_{1} m_{2}=0$, then

$$
\operatorname{ch}\left(\Gamma_{1}(\lambda)\right)=\frac{x_{1}^{m_{1}} x_{2}^{m_{2}}}{\left(1-x_{1} x_{2}\right)}, \quad \operatorname{ch}\left(\Gamma_{0}(\lambda)\right)=\frac{1}{\left(1-x_{1} x_{2}\right)} .
$$

(b) If $m_{1} m_{2} \neq 0$, then

$$
\begin{aligned}
\operatorname{ch}\left(\Gamma_{1}(\lambda)\right)=\frac{x_{1}^{m_{1}} x_{2}^{m_{2}}}{\left(1-x_{1} x_{2}\right)^{2}}\left\{1+\frac{A}{1-A}\left(1-A^{m_{2}}\right)+\frac{B}{1-B}\left(1-B^{m_{1}}\right)\right. \\
\left.+\frac{x_{1}^{3}}{1-x_{1}^{3}} A^{m_{2}}+\frac{x_{2}^{3}}{1-x_{2}^{3}} B^{m_{1}}\right\}
\end{aligned}
$$

and

$$
\operatorname{ch}\left(\Gamma_{0}(\lambda)\right)=\frac{1}{\left(1-x_{1} x_{2}\right)^{2}}\left\{-1+\frac{x_{1}^{3}}{1-x_{1}^{3}}+\frac{x_{2}^{3}}{1-x_{2}^{3}}\right\}
$$

Proof. (a) When $\lambda=\Lambda_{2}$, we have found $\operatorname{ch}\left(\Gamma_{k}(\lambda)\right.$ ), for $k$ in $\mathbf{Z}$, in (4.4). The cases in (a) follow by applying (4.3).

(b) In this case $\lambda$ is regular. To prove the formula for $\operatorname{ch}\left(\Gamma_{1}(\lambda)\right)$ one need only show, by Frobenius reciprocity, that $\operatorname{dim} V_{\nu}^{\nu}$ is given by the right-hand side of the desired equation. This may be accomplished by using the Kostant multiplicity formula (see [12, p. 131]; for the partition function for $S U(3)$ see [16, Table I]). For details, see [7]; the main theorem serves as a heuristic device to suggest the result and its method of proof (since an algorithm for tensor products of irreducibles is known (see [16])). Q.E.D.

\section{BIBLIOGRAPHY}

1. J. F. Adams, Lectures on Lie groups, Benjamin, New York, 1969. MR 40 \#5780.

2. L. Auslander and B. Kostant, Polarization and unitary representations of solvable Lie groups, Invent. Math. 14 (1971), 255-354. MR 45 \#2092.

3. J. G. T. Belinfante and B. Kolman, A survey of Lie groups and Lie algebras with applications and computational methods, SIAM, Philadelphia, 1973.

4. A. Borel and F. Hirzbruch, Characteristic classes and homogeneous spaces. I, Amer. J. Math. 80 (1958), 458-538. MR 21 \# 1586. 412.

5. C. Chevalley, Theory of Lie groups. I, Princeton Univ. Press, Princeton, N.J., 1946. MR 7,

6. L. Fonda and G. C. Ghirardi, Symmetry principles in quantum physics, Dekker, New York, 1970. 
7. J. Funderburk, Module structure of certain induced representations of compact Lie groups, Ph.D. Thesis, Univ. of Wisconsin, Madison, May, 1975.

8. J. E. Humphreys, Introduction to Lie algebras and representation theory, Springer-Verlag, New York, 1972. MR 48 \#2197.

9. S. Kobayashi and K. Nomizu, Foundations of differential geometry, Vol. II, Interscience, New York, 1969. MR 38 \#6501.

10. B. Kostant, Lie algebra cohomology and the generalized Borel-Weil theorem, Ann. of Math. (2) 74 (1961), 329-387. MR 26 \#265.

11. , Quantization and unitary representations, I. Prequantization, Lectures in Modern Analysis and Applications, III, Lecture Notes in Math., vol. 170, Springer-Verlag, Berlin, 1970, pp. 87-208. MR 45 \#3638.

12. H. Samelson, Notes on Lie algebras, Mathematical Studies, no. 23, Van Nostrand Reinhold, New York, 1969. MR 40 \# 7322.

13. J.-P. Serre, Represésentations linéaires et espaces homogènes kälériens des groupes de Lie compacts, Séminaire Bourbaki: 1953/54, Exposé 100, 2nd corr. ed., Secrétariat mathématique, Paris, 1959. MR 28 \# 1087.

14. J.-M. Souriau, Structure des systèmes dynamiques, Maitrises de Mathématiques, Dunod, Paris, 1970. MR 41 \#4866.

15. S. Sternberg, Lectures on differential geometry, Prentice-Hall, Englewood Cliffs, N.J., 1964. MR 33 \# 1797.

16. J. Tarski, Partition function for certain simple Lie algebras, J. Mathematical Phys. 4 (1963), 569-574. MR 26 \#5099.

17. Nolan R. Wallach, Harmonic analysis on homogeneous spaces, Dekker, New York, 1973.

Department of Mathematical Sciences, Northern Illinois University, DeKalb, Illinois 60115 